\title{
Mesenchymal stem cell-based developmental endothelial locus-1 gene therapy for acute lung injury induced by lipopolysaccharide in mice
}

\author{
YUN-FENG ZHAO $^{1}$, WEI XIONG ${ }^{1}$ and XUE-LING WU ${ }^{2}$ \\ ${ }^{1}$ Department of Respiratory Medicine, Pudong New Area, Gongli Hospital, Shanghai 200135; \\ ${ }^{2}$ Institute of Respiratory Medicine, Xinqiao Hospital, Third Military Medical University, Chongqing 400037, P.R. China
}

Received September 23, 2013; Accepted February 11, 2014

DOI: $10.3892 / \mathrm{mmr} .2014 .1988$

\begin{abstract}
Studies have suggested that bone marrow-derived mesenchymal stem cells (MSCs) may be used as a tool for gene therapy. Developmental endothelial locus-1 (Del-1) is a critical factor for cell migration and infiltration via the inhibition of the function of a major leukocyte adhesion receptor LFA-1 which prevents leukocyte adhesion to the endothelium. In the present study, we hypothesized that MSC-based Del-1 gene therapy may have potential therapeutic applications for lipopolysaccharide (LPS)-induced lung injury. The MSCs in the present assay were isolated from 6 week-old male mice. In order to investigate the therapeutic effect of the Del-1 gene on LPS-induced ALI mice, a lentivirus vector containing the Del-1 gene was constructed and transduced into the MSCs. In the in vivo assay, we induced lung injury with LPS injection and treated mice with different groups of MSCs, and compared with groups treated with MSCs alone, we observed that the administration with MSCs carrying Del-1 (MSCs-Del1) markedly alleviated the LPS-induced lung injury. There were significant decreases in the number of neutrophils in bronchoalveolar lavage (BAL) and the serum levels of TNF- $\alpha$ and IL-6 in the Del-1-expressed MSC-treated mice. Furthermore, compared with MSCs treated alone, Del1-MSC-treated mice also exhibited low lung injury scores, high protein concentrations and myeloperoxidase activity. In conclusion, treatment with Del-1-expressed MSCs significantly decreases the severity of endotoxin-induced acute lung injury and the level of inflammatory cytokines in mice.
\end{abstract}

Correspondence to: Dr Xue-Ling Wu, Institute of Respiratory Medicine, Xinqiao Hospital, Third Military Medical University, No. 183 Xinqiaozheng Street, Shapingba, Chongqing 400037, P.R. China

E-mail: wxueling@yeah.net

Key words: acute lung injury, mesenchymal stem cell, gene therapy

\section{Introduction}

Acute lung injury (ALI) and its more severe manifestation, acute respiratory distress syndrome (ARDS), are major complications in critically ill patients. The mortality rate of ARDS/ALI remains high due to the lack of effective treatments (1). ALI is a syndrome of widespread lung inflammation and increased pulmonary vascular permeability resulting in pulmonary edema and hypoxia, and may contribute to multiple organ failure and death. ALI is most commonly caused by sepsis, pneumonia, trauma or aspiration of the gastric contents. Infection is also the most common cause of ALI and is associated with a higher mortality rate than non-infectious $\operatorname{ALI}(2,3)$. The inflammatory process is important in the pathogenesis of infectious and non-infectious ALI, and the degree of acute inflammation is strongly correlated with outcome (4).

Recently, transplantation of various stem or progenitor cells including bone marrow-derived mesenchymal stem cells (MSCs) or endothelial progentior cells, has been reported to reduce mortality and attenuate ALI induced by endotoxins or sepsis in a rodent model (5-7). Bone marrow-derived MSCs retain the ability to differentiate into a distinct variety of cell lineages (8), including chondrocytes, myoblasts, endothelial cells, epithelial cells and neurocytes, and have self-renewal capacities. There is an increased interest in understanding the biology of MSCs for potential clinical use as cell-based therapies $(9,10)$. Recent in vivo and in vitro studies indicate that multi-potent mesenchymal stromal or stem cells may also have therapeutic effects on ALI (9,11-14).

Del-1 is a $52 \mathrm{kDa}$ glycoprotein secreted by endothelial cells, which associates with the endothelial cell surface and extracellular matrix (15-17). Human Del-1 shares $>97 \%$ amino acid identity with the mouse counterpart; the expression of Del-1 was initially observed in embryonic cells including in the endothelium and thymus, and subsequent subsets of macrophages and hypertrophic chondrocytes. However, it was recently reported that Del-1 is expressed in adult mice in a tissue-specific manner, including high expression in the lung, brain and eye, weakly in the kidney and absent in the intestine, liver, heart, spleen, whole blood and bone marrow cells (18). It was reported that Del-1 is a ligand for LFA-1 and that Del-1 actually inhibits the function of LFA-1. Under physiological flow conditions, addition of Del-1 to 
the system where leukocytes adhere onto immobilized ICAM-1, via interactions with active LFA-1, represses the adhesion of leukocytes (18). Del-1-mediated inhibition of the interaction between LFA-1 and ICAM-1 further implies that Del-1 may be used as a therapeutic tool for inflammatory diseases.

The aim of the present study was to examine the effect of Del-1 overexpressed-MSCs treatment on the ALI mouse model that was induced by lipopolysaccharide (LPS) injection. MSCs were isolated from the bone marrow of C57/B6 mice and expanded in vitro prior to administration through the injection of the tail vein. The cDNA of Del-1 were cloned from mouse lung tissue. The data suggested that when over-expressed by retroviral transduction, the treatment of Del-1 over-expressed MSCs have beneficial effects on LPS-induced ALI, including lower neutrophils and proinflammatory cytokines concentration in mice bronchoalveolar lavage (BAL). Our data demonstrate a potential new approach to the treatment of ALI.

\section{Materials and methods}

Mice. Thirty-six C57/B6 mice were purchased from the SLRC laboratory (Shanghai, China). The mice were randomized into control group ( $n=10$, phosphate-buffered saline (PBS) treated), ALI group ( $n=10$, LPS treated only), MSCs control group $(n=10$, LPS+MSCs-GFP treated) and MSCs-Del-1 treated group ( $n=10)$. Mice were sacrificed at $24 \mathrm{~h}$ following LPS and MSC administration. The study was approved by the Ethics Committee of Third Military Medical University (Chongqing, China).

Establishment of the ACI model of mice. Female C57/B6 mice (8-10 weeks old) were treated with either $20 \mathrm{mg} / \mathrm{kg}$ LPS (Sigma-Aldrich, St. Louis, MO, USA) from Escherichia coli (serotype 0111:B4) in $100 \mu \mathrm{l}$ PBS or an equal volume of PBS, as a vehicle control, by intraperitoneal injection.

MSC isolation and culture. Fresh bone marrow cells were harvested from the femurs of six-weeks-old male mice by flushing with DMEM (Thermo Fisher Scientific, Inc., Waltham, MA, USA) supplemented with $15 \%$ fetal bovine serum (FBS; Beijing Solarbio Science \& Technology Co., Ltd., Beijing, China). The bone marrow mononuclear cells were isolated by density gradient centrifugation (Ficoll-Paque PLUS; Pharmacia \& Upjohn Company, Peapack, NJ, USA) and cultured in low-glucose DMEM containing 15\% FBS and 1\% penicillin/ streptomycin (Beijing Solarbio Science \& Technology Co., Ltd.) at $37^{\circ} \mathrm{C}$ in a humidified incubator with $5 \% \mathrm{CO}_{2}$. The nonadherent cells were removed by medium changes. The cells were passaged every 3-4 days by trypsinization and the fourth passage cells were used for in vivo experiments.

Lentivirus preparation and infection. Vectors for lentivirus packaing including pMDL, pRev pVSVG and lentivirus transfer vector were purchased from Clontech Laboratories (Mountain View, CA, USA). Lentiviral vectors were transfected into 293T cells using Lipofectamine (Invitrogen Life Technologies, Carlsbad, CA, USA). Following $24 \mathrm{~h}$, the medium was replaced with DMEM containing $15 \%$ FBS. A total of $48 \mathrm{~h}$ post-transfection, the lentiviral supernatants were harvested, supplemented with $6 \mu \mathrm{g} / \mathrm{ml}$ polybrene and used to infect MSC cells.
MSC administration. A total of $1 \mathrm{~h}$ following LPS injection, the mice were given either $5 \times 10^{6}$ MSCs in $100 \mu \mathrm{l}$ PBS (in the MSCs-treated ALI group) or $100 \mu 1$ PBS (in the PBS-treated ALI and control groups) via tail vein injection. At $24 \mathrm{~h}$ following the injection, samples were collected from each mouse for assessment of lung injury.

Histopathological analysis and lung injury scores. Following sacrifice, at each time point the whole left lower lobe of the lung was fixed in a $4 \%$ formaldehyde neutral buffer solution for $24 \mathrm{~h}$, dehydrated in a graded ethanol series, embedded in paraffin and sliced at $5 \mu \mathrm{m}$. Paraffin sections were stained with hematoxylin and eosin $(\mathrm{H} \& \mathrm{E})$ for histopathological analysis.

In order to assess the severity of the lung damage, a semiquantitative histological index of quantitative assessment (IQA) of lung injury was utilized. Eight sections were randomly selected from each group of rats and ten fields from each section were examined by microscopy (magnification, x40). A pathologist evaluated all of the sections in a blinded manner. The average values of the lung injury obtained were considered a semi-quantitative histological IQA of lung injury.

Quantitative (q)PCR analysis. Total RNA was extracted from pulmonary tissues using TRIzol reagent (Invitrogen Life Technologies) according to the manufacturer's instructions. RT-PCR kits (Takara Healthcare, Inc., Kyoto, Japan) were used for the qPCR experiment. Total RNA template $(4 \mu \mathrm{g})$ were used to construct the cDNA by using AMV reverse transcriptase and random 9-mers as the first-strand primer. Synthesized cDNA was used in qPCR experiments. qPCR was performed using a 40-cycle two-step PCR with sequence-specific primer pairs using the ABI7900 fast real-time detection system (Invitrogen Life Technologies). Primers were designed using the Primer Express 3.0 software. The level of the mRNA expression was evaluated as a ratio based on $\mathrm{qPCR}$ results for lung tissue GAPDH mRNA.

ELISA. Detection of TNF- $\alpha$ and IL-6 was conducted using ELISA according to the manufacturer's instructions. The experiment was repeated three times and the results expressed with the mean value.

Lung wet/dry weight ratio. The superior lobe of the right lung was cleansed and weighed to obtain the wet weight, and was then place in an oven at $80^{\circ} \mathrm{C}$ for $48 \mathrm{~h}$ for measurement of the dry weight. The ratio of the wet weight to dry weight was calculated to assess the tissue edema.

Bronchoalveolar lavage (BAL) examination. The trachea was exposed and cannulated with a catheter. The left lung was lavaged three times with sterile PBS in a volume of $0.5 \mathrm{ml} /$ wash. The fluid recovered following lavage was $>90 \%$ on average. The BAL fluid (BALF) was centrifuged at $700 \mathrm{x} \mathrm{g}$ for $10 \mathrm{~min}$ at $4^{\circ} \mathrm{C}$ and the supernatant was stored at $-80^{\circ} \mathrm{C}$ for cytokine and protein analysis, while the cell pellet was resuspended in PBS for counting the neutrophils.

Myeloperoxidase (MPO) activity assay. MPO activity in the homogenized lung tissue was measured as previously described by Gray et al (19). The MPO concentration was detected using 


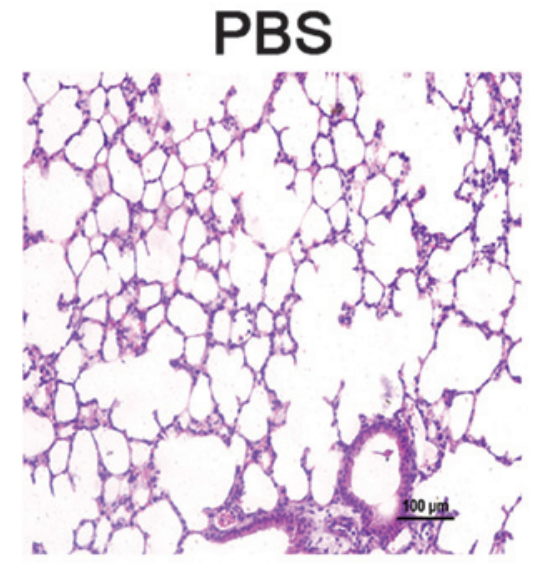

B

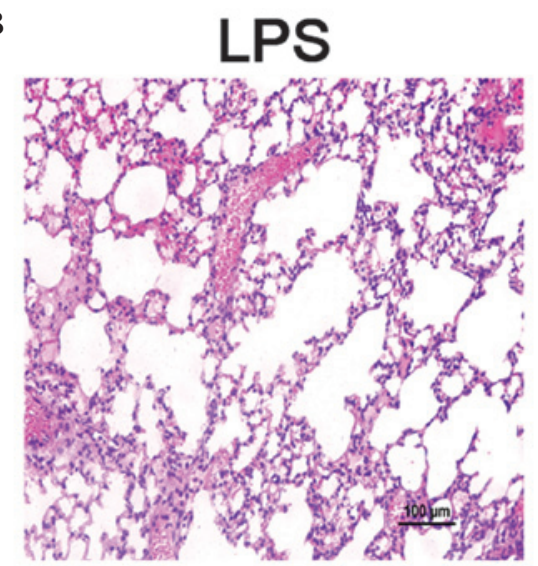

C

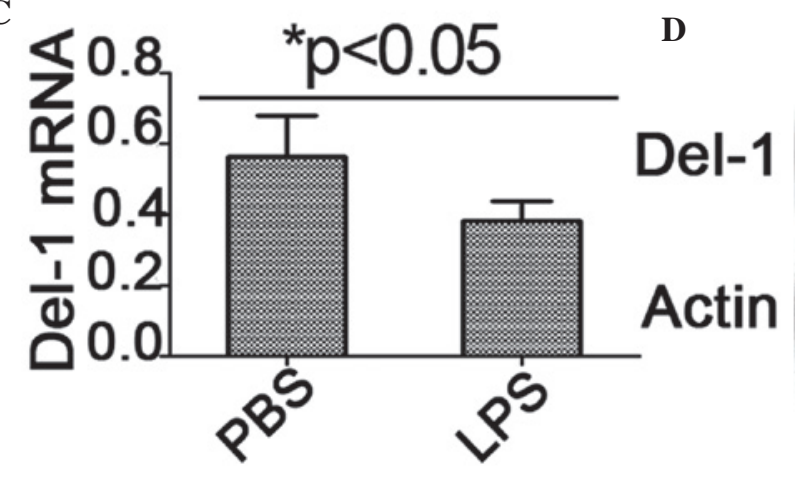

LPS PBS

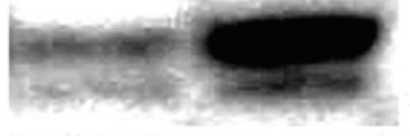

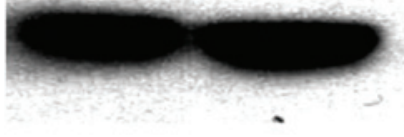

Figure 1. Induction of ALI in mice and expression of Del-1 in different ALI mice lung tissues. The H\&E-stained lung sections following 24 h of induction of ALI in the (A) PBS control mice and (B) LPS-induced ALI mice. (C) Level of Del-1 mRNA in different ALI mice groups ("P<0.05 compared with PBS control). (D) Level of Del-1 protein in different ALI mice groups. Data are expressed as the mean \pm SD (error bars). ALI, acute lung injury; Del-1, developmental endothelial locus-1; PBS, phosphate-buffered saline; LPS, lipopolysaccharide; H\&E, hematoxylin and eosin.

a MPO ELISA kit (Blue Gene Biotech, Shanghai, China). Briefly, the lung tissues were homogenized and centrifuged at $15,000 \mathrm{x} \mathrm{g}$ for $20 \mathrm{~min}$ at $4^{\circ} \mathrm{C}$. The supernatants and standard sample were added into a microtiter plate (100 $\mu \mathrm{l} / \mathrm{well})$ precoated with a murine anti-MPO $\mathrm{mAb}$. Following incubation for $1 \mathrm{~h}$ at $37^{\circ} \mathrm{C}$, the plate was washed six times followed by the addition of the substrate and stop solution, and the optical density (OD) at $450 \mathrm{~nm}$ was measured using a microplate reader. All the samples were assayed in triplicate.

Measurement of protein concentration in lung BALF. The concentration of protein in the BALF was measured using Bradford reagent and a protein assay kit (Bio-Rad, Hercules, CA, USA). Briefly, $160 \mu 1$ of each standard and sample solution was pipetted into separate microtiter plate wells and $40 \mu \mathrm{l}$ of the dye reagent was added to each well and mixed thoroughly. The mixture was incubated at room temperature for at least 5 min prior to the measurement of the OD at $595 \mathrm{~nm}$. Comparison to a standard curve provided a relative measurement of the protein concentration.

Statistical analysis. All the data were analyzed using SPSS 13.0 software (SPSS, Inc., Chicago, IL, USA) and expressed as the mean \pm SD. Significant differences were assessed by one-way analysis of variance (ANOVA) followed by Fisher protected least significant difference test. $\mathrm{P}<0.05$ was considered to indicate a statistically significant difference.

\section{Results}

Increased expression of Del-1 in LPS-induced ACI. Previous studies have demonstrated that, as an inhibitor of cell migration, Del-1 inhibits the migration of a variety of different cells, particularly the process of immune cell migration through the blood vessel wall to the lesion site. In order to study the function of Del-1 in ACI, an ACI in vivo mouse model was established by intravenous endotoxin injection and the lung tissue of the different groups of mice were obtained for paraffin section. As demonstrated in Fig. 1, microscopic examination of the lung following $\mathrm{H} \& \mathrm{E}$ staining revealed exudative changes, patchy hemorrhage, a thickened alveolar interstitium and heavy infiltration of inflammatory neutrophils and lymphocytes into the intra-alveolar and interstitial spaces in the lungs of mice with LPS-induced ALI. To further assess the level of Del-1 in the injured lung tissue, the expression of Del-1 in different mice lung tissue samples was detected by qPCR and western blot analysis. It was identified that the level of Del-1 mRNA and protein was decreased compared with the health control group (Fig. 1B).

MSC characterization and retrovirals induces Del-1 overexpression in MSCs. Previous studies have demonstrated that the MSCs have therapeutic effects on LPS-induced ALI mice, in addition, the Del-1 may inhibit the migration of pro-inflammatory cells to the lesion. Therefore, whether the Del-1 overexpressed MSCs have an optimal effect on ALI was 
A
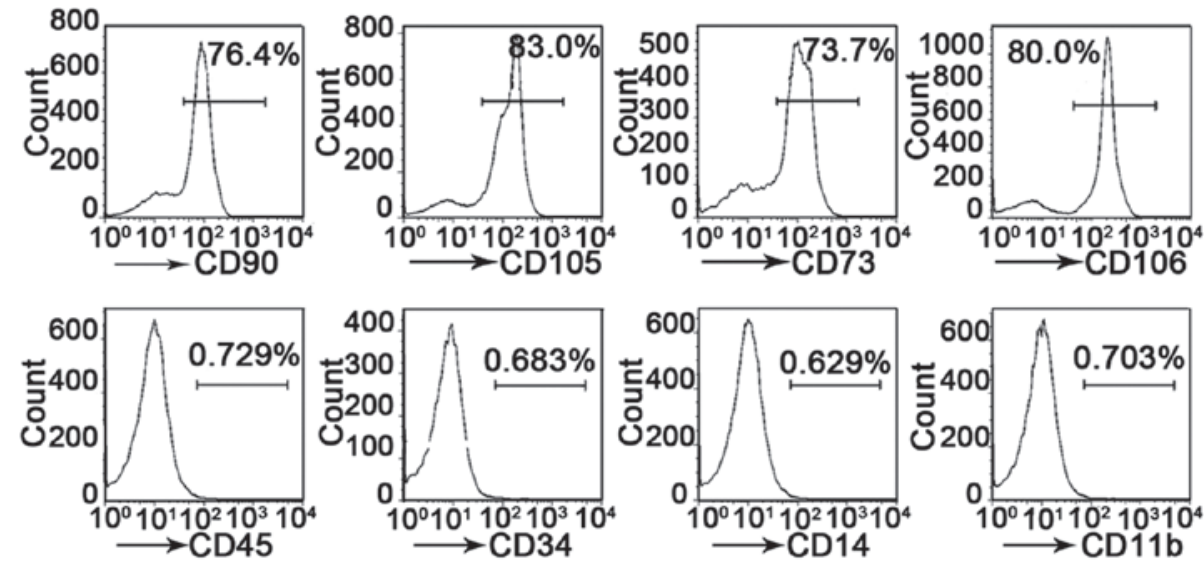

B

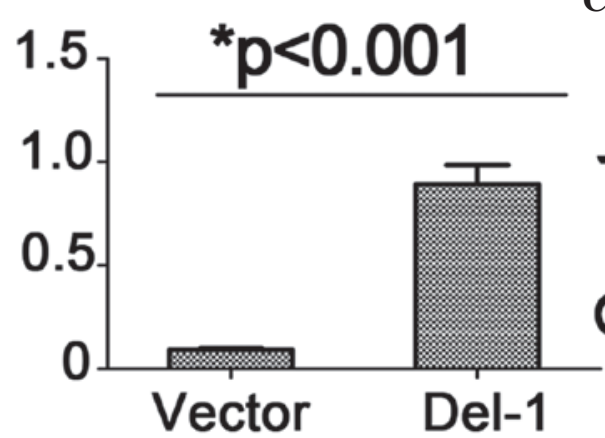

C

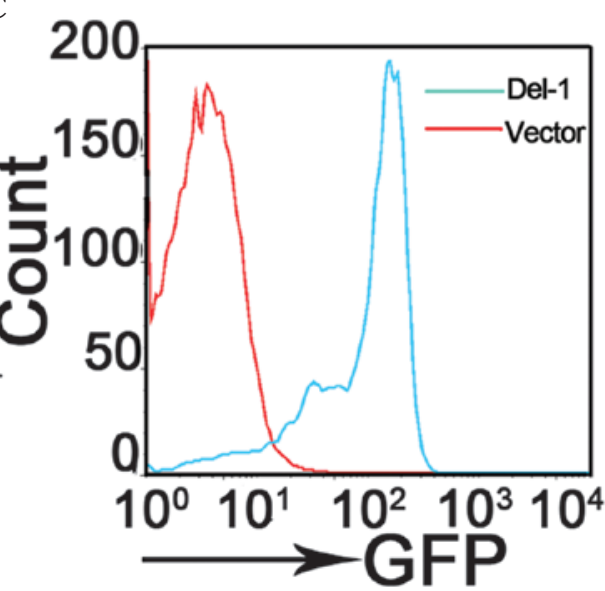

Figure 2. Flow cytometric immunophenotype analysis of the MSCs and the expression of Del-1 in transduced MSCs using a lentivirus vector. (A) The adherent MSCs expressed CD90, CD105, CD73 and CD106, but not CD45, CD34, CD14 and CD11b. (B) The level of Del-1 mRNA and protein in lentivirus-infected MSCs. (C) The transduction of Del-1 into MSCs using lentivirus vector. MSCs, mesencyhmal stem cells; Del-1, developmental endothelial locus-1.

investigated. Bone marrow-derived MSCs were isolated from the mice and flow cytometric immunophenotype analysis revealed that almost all of these cells expressed the typical mesenchymal stem cell surface markers CD105, CD90, CD73 and CD106, but not the hematopoietic lineage markers CD45, CD34, CD14 and CD11b (Fig. 2A).

To investigate the therapeutic effect of Del-1 overexpressed MSCs on ALI, a Del-1 expressing lentiviral vector was generated and MSCs cells were infected. Overexpression of Del-1 mediated by lentiviral transduction was confirmed in GFP cells by FACS sorting (Fig. 2C), the level of Del-1 mRNA in MSC cell was detected by qPCR (Fig. 2B). The data suggested that the Del-1 expressing lentiviral vector increased the expression level of Del-1 in bone marrow-derived MSCs.

Effect of different treated MSC transplantation on lung histopathology and lung injury scores. As demonstrated in Fig. 3 , the transplantation of MSCs markedly reduced the infiltration of inflammatory cells and improved the histological features of the lung, $24 \mathrm{~h}$ following injection via the tail vein (Fig. 3C). The transplantation of a Del-1-MSCs had a distinct therapeutic effect on LPS-induced ALI compared with the MSCs treated group (Fig. 3D).

To further assess the degree of pulmonary damage, injury in the left lung was scored in each group (Fig. 3E). The lung injury index reached the highest value $24 \mathrm{~h}$ following transplantation and the scores of the MSCs and Del-1-MSCstreated groups were significantly lower than those of the PBS-treated control group $(\mathrm{P}<0.001)$.

The neutrophil count in BALF and the lung wet/dry ratio are revealed in Fig. 3F and $\mathrm{H}$. The level of lung wet/dry ratio and neutrophil count decreased significantly in the MSC-treated group and Del-1-expressed MSC-treated group mice compared with the controls. In addition, the administration of the Del-1expressed MSCs also reduced the neutrophil counts in BALF and the lung wet/dry ratio, and the difference in cell count and wet/dry ratio between MSCs treated group and Del-1 treated group were significant $(\mathrm{P}<0.05)$.

Additionally, we detected the mRNA level of Del-1 in the lung tissue of the MSC-treated groups of mice. We demonstrated that the expression of Del-1 was increased in the Del-1-expressed MSCs treated mice and this data was consistent with the result of the therapeutic effect.

IL-6, TNF- $\alpha$ and protein concentration in BALF and MPO in lung homogenates. The expression levels of TNF- $\alpha$ and IL-6 in BALF were significantly increased in PBS-treated ALI group compared with those in the healthy control group. The MSCs or Del-1-expressed MSC treatment significantly reduced the elevated TNF- $\alpha$ and IL- 6 levels. Furthermore, the level of TNF- $\alpha$ in BALF was significantly decreased in Del-1 expressed MSC-treated group compared with those 

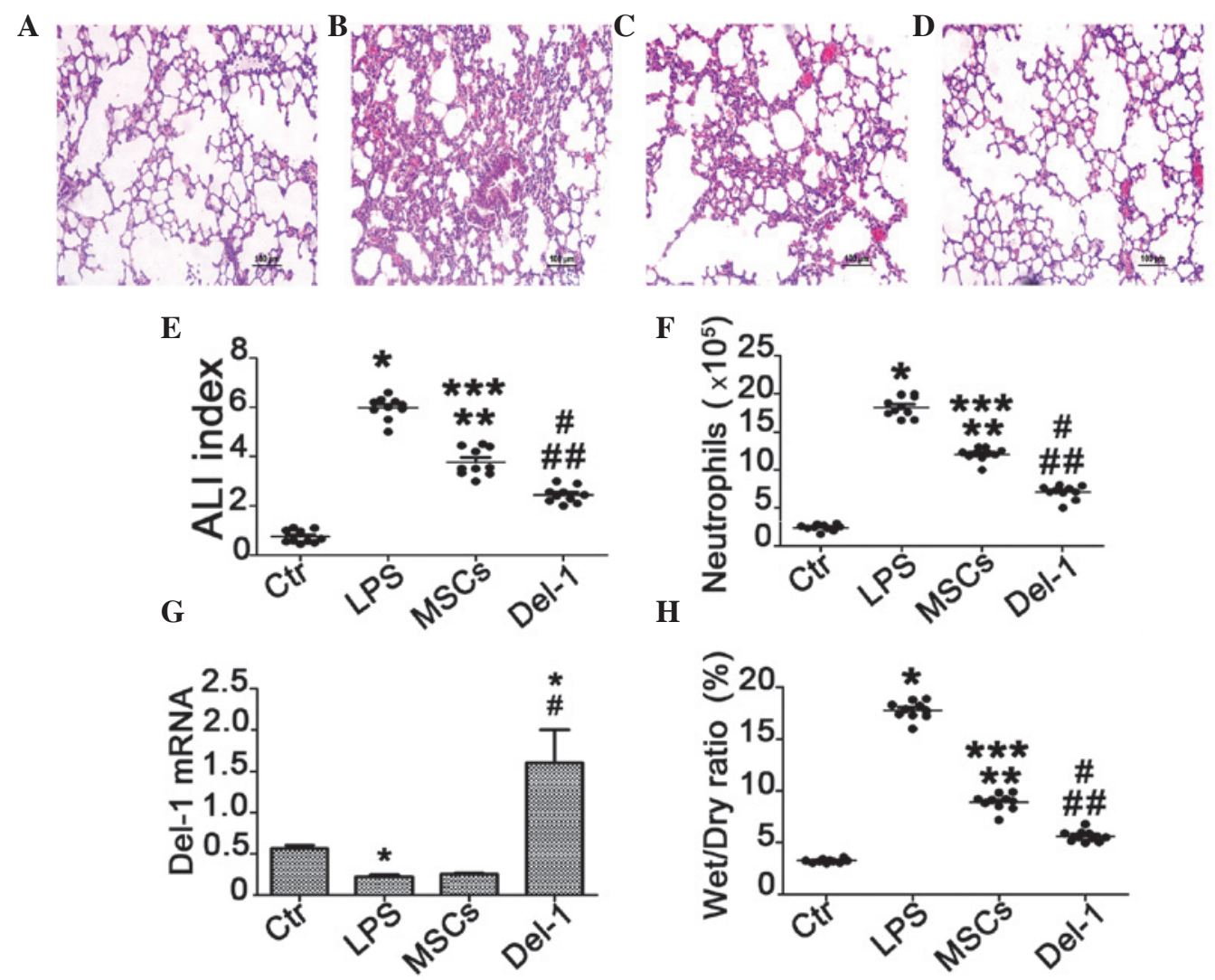

Figure 3. Histopathological index of injured mice lungs. The H\&E-stained lung sections following the different treatments in (A) healthy controls; (B) PBS treated group; (C) MSC-treated only group and (D) Del-1-MSC treated group. (E) The lung injury index of the different treated mice. (F) The neutrophil count in the BALF of different treated mice. (G) The level of Del-1 mRNA in different treated mice. (H) The wet/dry ratio of different groups of mice. "P<0.05 compared with the Ctr group mice; ${ }^{* *} \mathrm{P}<0.05$ compared with LPS group mice; ${ }^{* * *} \mathrm{P}<0.05$ compared with the Ctr group mice; ${ }^{*} \mathrm{P}<0.05$ compared with the LPS group mice; ${ }^{\# \#} \mathrm{P}<0.05$ compared with the MSC group mice. ALI, acute lung injury; Del-1, developmental endothelial locus-1; PBS, phosphate-buffered saline; LPS, lipopolysaccharide; H\&E, hematoxylin and eosin; BALF, bronchoalveolar lavage fluid; MSCs, mesencyhmal stem cells; Ctr, control.

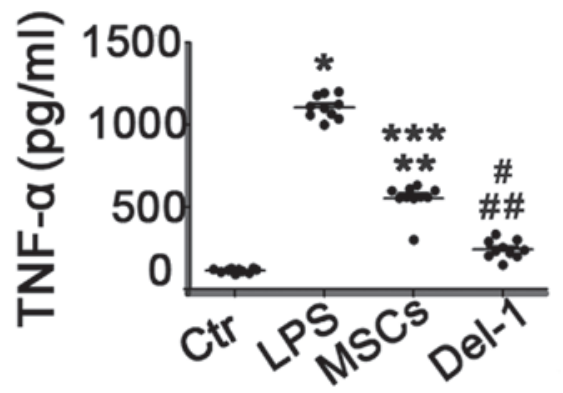

C

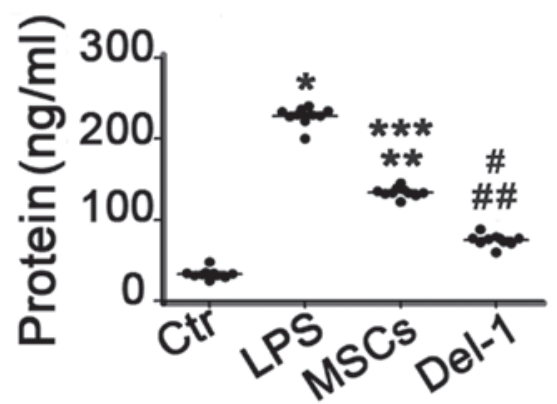

B

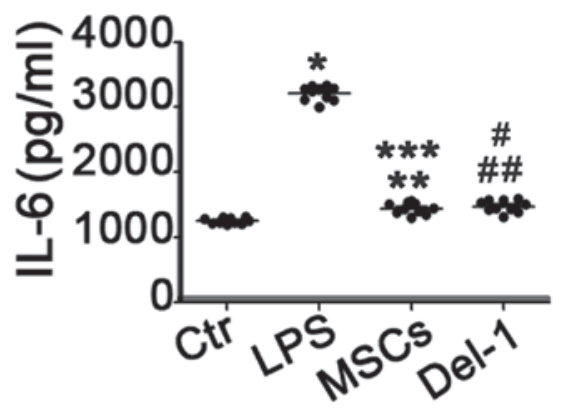

D

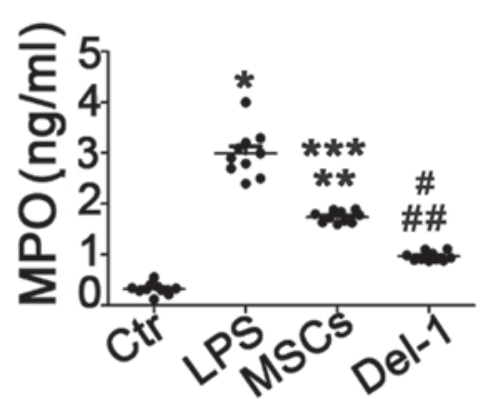

Figure 4. Proinflammatory cytokines, protein and MPO concentration in BALF of ACI mice lung homogenates. (A) TNF- $\alpha$; (B) IL-6; (C) protein and (D) MPO concentration in BALF of ALI mice following the different types of treatment." $\mathrm{P}<0.05$ compared with the Ctr group mice; ${ }^{* * *} \mathrm{P}<0.05$ compared with the LPS group mice; ${ }^{* * *} \mathrm{P}<0.05$ compared with the $\mathrm{Ctr}$ group mice; ${ }^{\#} \mathrm{P}<0.05$ compared with the LPS group mice; ${ }^{\# \#} \mathrm{P}<0.05$ compared with the MSCs group mice. ALI, acute lung injury, Del-1, developmental endothelial locus-1; PBS, phosphate-buffered saline; LPS, lipopolysaccharide; H\&E, hematoxylin and eosin; BALF, bronchoalveolar lavage fluid; MPO, myeloperoxidase; MSC, mesencyhmal stem cells; Ctr, control. 
in the MSC-treated group, however there was no change in the concentration of IL- 6 between MSC-treated group and Del-1 expressed MSC-treated group. In addition, the protein concentration in the BALF and the level of MPO in the lung homogenates were consistent with the results of the cytokine level in BALF (Fig. 4C and D).

\section{Discussion}

As demonstrated in previous animal studies, bone marrow-derived MSCs are able to alleviate LPS-induced ACI by restoring lung function and increasing survival rate via its anti-inflammatory, anti-apoptotic and immune regulatory properties and may therefore provide a novel therapy for ALI. Recently, it was demonstrated that Del-1 functions as an endogenous inhibitor of a major leukocyte adhesion receptor, LFA-1, to prevent leukocyte adhesion to the endothelium (18). Unlike many adhesion molecules, including selectins and Ig superfamily members promoting leukocyte adhesion on the endothelium, Del-1 inhibits the process of leukocyte binding to the endothelium, thereby suppressing entrance of leukocytes to inflamed tissues.

Our data indicate that the administration of Del-1 modified MSCs after LPS induction protects lung tissue from injury by significantly reducing the extent of inflammation, although only a limited effect was observed following the administration of MSCs alone. Additionally, our in vivo data suggested that the histopathology of lung injury was greatly improved following the administration of MSCs carrying the Del-1 gene compared with the treatment with MSCs alone.

A number of studies have suggested that bone-marrowderived MSCs $(9,20-22)$ or Del-1 $(18,23,24)$ have therapeutic applications in acute lung injury. To further define the therapeutic potential of MSCs, we used a lentivirus vector containing the Del-1 gene to infect the MSCs, then treated ALI mice with these Del-1 expressed MSCs. Our results suggested that the MSCs were able to have a therapeutic effect, as well as act as gene delivery vehicles.

In the present study, the cells that were utilized for ALI therapy were isolated from the bone marrow. These cells exhibited several typical characteristics of MSCs. As plasticadherent cells, these bone marrow-derived MSCs express surface markers typically associated with MSCs but that are not hematopoietic stem cell markers. The MSCs differentiated from bone marrow were analyzed by flow cytometric immunophenotype, and it was identified that almost all these cells expressed the cell surface markers CD105, CD90, CD73 and CD106, but not the hematopoietic lineage markers CD45, CD34, CD14 and CD11b (Fig. 2).

Lentiviral vectors were selected to modify MSCs with the Del-1 gene, due to their high efficiency in gene delivery and ability to integrate the gene into the MSCs during cell division (18). The results demonstrated that high gene transduction efficiency was achieved at day 5 following exposure to lentiviral vectors, suggesting gene integration was successful (Fig. 2).

It has been reported that Del-1 is a ligand for LFA-1, and that Del-1 is able to inhibit the function of LFA-1. Under physiological flow conditions, the addition of Del-1 to the system where leukocytes adhere onto immobilized ICAM-1 via interaction with active LFA-1, represses the adhesion of leukocytes (24). In the present study, the over-expression of Del-1 in the recipient lung was achieved by MSC-based gene delivery (Fig. 2B). It was demonstrated that MSC-based gene therapy attenuates the inflammatory reaction and vascular leakage in the lung following LPS exposure. Furthermore, it was also able to improve lung histopathological changes. These beneficial effects may be mediated by the downregulation of pro-inflammatory gene expression, including TNF- $\alpha$ and IL-6 (Fig. 4).

In summary, our data indicate that Del-1-carrying MSC therapy may ameliorate the injurious effects of LPS-induced acute lung injury. As an effective cellular vehicle for the treatment of lung disease, MSCs and Del-1 may provide a platform for designing novel attractive therapeutic modalities to reduce tissue injury in lung injury disease.

\section{Acknowledgements}

The authors thank all subjects enrolled in this study. The present study was supported by grants from the Pudong New Area health bureau Foundation of Shanghai (grant no. PWRd 2012-07 to Yun-feng Zhao) and the National Natural Science Foundation of China (grant no. 81070056 and 81270130 to Xue-ling $\mathrm{Wu})$.

\section{References}

1. Rubenfeld GD, Caldwell E, Peabody E, Weaver J, et al: Incidence and outcomes of acute lung injury. N Engl J Med 353: 1685-1693, 2005.

2. Sheu CC, Gong MN, Zhai R, et al: Clinical characteristics and outcomes of sepsis-related vs non-sepsis-related ARDS. Chest 138: 559-567, 2010.

3. Hu X, Qian S, Xu F, et al: Incidence, management and mortality of acute hypoxemic respiratory failure and acute respiratory distress syndrome from a prospective study of Chinese paediatric intensive care network. Acta Paediatr 99: 715-721, 2010.

4. Ware LB, Koyama T, Billheimer DD, et al: Prognostic and pathogenetic value of combining clinical and biochemical indices in patients with acute lung injury. Chest 137: 288-296, 2010.

5. Cribbs SK, Matthay MA and Martin GS: Stem cells in sepsis and acute lung injury. Crit Care Med 38: 2379-2385, 2010.

6. Lee JW, Gupta N, Serikov V and Matthay MA: Potential application of mesenchymal stem cells in acute lung injury. Expert Opin Biol Ther 9: 1259-1270, 2009.

7. Matthay MA, Goolaerts A, Howard JP and Lee JW: Mesenchymal stem cells for acute lung injury: preclinical evidence. Crit Care Med 38 (Suppl): S569-S573, 2010.

8. Das R, Jahr H, van Osch GJ and Farrell E: The role of hypoxia in bone marrow-derived mesenchymal stem cells: considerations for regenerative medicine approaches. Tissue Eng Part B Rev 16: 159-168, 2010.

9. Rojas M, Xu J, Woods CR, et al: Bone marrow-derived mesenchymal stem cells in repair of the injured lung. Am J Respir Cell Mol Biol 33: 145-152, 2005.

10. Krause DS, Theise ND, Collector MI, et al: Multi-organ, multilineage engraftment by a single bone marrow-derived stem cell. Cell 105: 369-377, 2001.

11. Krause DS: Bone marrow-derived cells and stem cells in lung repair. Proc Am Thorac Soc 5: 323-327, 2008.

12. Gupta N, Su X, Popov B, Lee JW, Serikov V and Matthay MA: Intrapulmonary delivery of bone marrow-derived mesenchymal stem cells improves survival and attenuates endotoxin-induced acute lung injury in mice. J Immunol 179: 1855-1863, 2007.

13. Xu J, Woods CR, Mora AL, et al: Prevention of endotoxin-induced systemic response by bone marrow-derived mesenchymal stem cells in mice. Am J Physiol Lung Cell Mol Physiol 293: L131-L141, 2007.

14. Xu J, Qu J, Cao L, et al: Mesenchymal stem cell-based angiopoietin-1 gene therapy for acute lung injury induced by lipopolysaccharide in mice. J Pathol 214: 472-481, 2008. 
15. Phillipson M, Heit B, Colarusso P, Liu L, Ballantyne CM and Kubes P: Intraluminal crawling of neutrophils to emigration sites: a molecularly distinct process from adhesion in the recruitment cascade. J Exp Med 203: 2569-2575, 2006.

16. Schenkel AR, Mamdouh Z and Muller WA: Locomotion of monocytes on endothelium is a critical step during extravasation. Nat Immunol 5: 393-400, 2004.

17. Barreiro O, Yanez-Mo M, Serrador JM, et al: Dynamic interaction of VCAM-1 and ICAM-1 with moesin and ezrin in a nove endothelial docking structure for adherent leukocytes. J Cell Biol 157: 1233-1245, 2002.

18. Choi EY, Chavakis E, Czabanka MA, et al: Del-1, an endogenous leukocyte-endothelial adhesion inhibitor, limits inflammatory cell recruitment. Science 322: 1101-1104, 2008.

19. Gray KD, Simovic MO, Chapman WC, et al: Endotoxin potentiates lung injury in cerulein-induced pancreatitis. Am J Surg 186: 526-530, 2003.
20. Aslam M, Baveja R, Liang OD, et al: Bone marrow stromal cells attenuate lung injury in a murine model of neonatal chronic lung disease. Am J Respir Crit Care Med 180: 1122-1130, 2009.

21. Ortiz LA, Gambelli F, McBride C, et al: Mesenchymal stem cell engraftment in lung is enhanced in response to bleomycin exposure and ameliorates its fibrotic effects. Proc Natl Acad Sci USA 100: 8407-8411, 2003.

22. van Haaften T, Byrne R, Bonnet S, et al: Airway delivery of mesenchymal stem cells prevents arrested alveolar growth in neonatal lung injury in rats. Am J Respir Crit Care Med 180: 1131-1142, 2009.

23. Basit A, Reutershan J, Morris MA, Solga M, Rose CE Jr and Ley K: ICAM-1 and LFA-1 play critical roles in LPS-induced neutrophil recruitment into the alveolar space. Am J Physiol Lung Cell Mol Physiol 291: L200-L207, 2006.

24. Choi EY: Inhibition of leukocyte adhesion by developmental endothelial locus-1 (del-1). Immune Netw 9: 153-157, 2009. 